\title{
Lack of Utility of Cytokeratins in Differentiating Pseudocarcinomatous Hyperplasia of Granular Cell Tumors from Oral Squamous Cell Carcinoma
}

\author{
Kamal Al-Eryani ${ }^{1,2 *}$, Jumana Karasneh ${ }^{1,3}$, Parish P Sedghizadeh ${ }^{1}$, Saravanan \\ Ram $^{1}$, Faleh Sawair ${ }^{4}$
}

\begin{abstract}
Granular cell tumor (GCT) of the oral cavity is a benign lesion. Half of oral GCTs demonstrate pseudocarcinomatous hyperplasia (PCH) of the mucosa which can mimic invasive islands of oral squamous cell carcinoma (SCC). Such similarity can be confusing when diagnosing or evaluating the two conditions, potentially leading to misdiagnosis or misclassification. Indeed, several misdiagnosed cases of oral GCT have been reported in the literature as OSCC or malignant oral GCT that resulted in unnecessary aggressive treatment for the affected patients. The aim of this study was to investigate if the cytokeratin pattern of the PCH can help in differentiating GCT from oral SCC. To distinguish between these two entities, we examined 12 patient specimens of oral GCT-PCH and oral SCC histologically and via immunohistochemistry (IHC) for CK13, CK17 and P75. The results suggest that the cytokeratin profile of $\mathrm{PCH}$ is similar to that of oral SCC. Therefore, consideration of IHC findings for epithelial markers alone may lead to erroneous diagnosis; thus, the presence of the granular tumor underneath the PCH and its immunopositivity for P75 or other neural definition markers can be essential to identify the underlying tumor and exclude oral SCC. Finally we recommend more studies on the molecular biology of PCH to understand how it can mimic oral SCC histologically without harboring its malignant phenotype clinically, which could have significant translational potential for understanding invasive oral SCC.
\end{abstract}

Keywords: Granular cell tumor - pseudoepitheliomatous hyperplasia - pseudocarcinomatous hyperplasia - Squamous

Asian Pac J Cancer Prev, 17 (4), 1785-1787

\section{Introduction}

Granular cell tumor (GCT) is a benign lesion. It can occur in almost any part of the human body such as the oral cavity, breast, large intestine and stomach (Lack, et al. ,1980; Patti et al., 2006; Huyskens and Geniets 2014; Zhang et al., 2014). For both GCT and oral squamous cell carcinoma (OSCC), the most common growth site in the oral cavity is the tongue (Neville, 2009). Furthermore, they may share epithelial histopathological criteria and failure to identify the histopathological differences between them can result in a misdiagnosis of OSCC, particularly in small biopsies that sample only part of the lesion (Tan et al., 2013).

Although tumor cells in GCT lack cytological atypia or dysplasia of surface epithelium, $50 \%$ of intraoral GCTs demonstrate pseudocarcinomatous hyperplasia (PCH) - also called pseudoepitheliomatous hyperplasia of overlying oral epithelium- which can mimic OSCC.
Indeed, several misdiagnosed cases of GCT have been reported in the literature (Wolber et al., 1991; AbuEid and Landini 2006) that resulted in unnecessary invasive treatment. To help distinguish between the two entities, many studies have been done to establish the immunohistochemical profile of GCT. These studies suggested numerous antibodies stains such as S-100 protein, neuron-specific enolase and myelin proteins, inhibin- $\alpha$ and P75 to stain GCT (Vered et al. 2009; Freitas et al.,2012). The aim of this study was to investigate if the cytokeratin pattern of the PCH can help in differentiating GCT from oral SCC.

\section{Materials and Methods}

\section{Materials}

A total of 12 surgical specimens, 3 of GCT and 9 oral SCC, were selected from Oral Pathology cases, Department of Oral Medicine, Pathology and Radiology,

${ }^{1}$ Oral Medicine and Orofacial Pain Center, University of Southern California, Los Angeles, California USA, ${ }^{2}$ Department of Oral Medicine and Pathology, Sana' a University, Yemen, ${ }^{3}$ Department of Oral Medicine and Oral Surgery, Jordan University of Science and Technology, Irbid, ${ }^{4}$ Department of Oral and Maxillofacial Surgery, Oral Medicine, Oral Pathology and Periodontology, Faculty of Dentistry, The University of Jordan, Amman, Jordan*For correspondence: aleryani@usc.edu 
Faculty of Dentistry, Sana'a, Yemen. The surgical specimens were fixed in $10 \%$ formalin and routinely embedded in paraffin. One set of $5 \mu \mathrm{m}$ serial sections was stained with hematoxylin and eosin (H\&E) and used to re-evaluate the pathologic diagnosis. The other set was used for immunohistochemical investigations with the antibodies described below.

\section{Antibodies}

Mouse monoclonal antibodies against human $\mathrm{K}$ (keratin), K13, K17 were purchased from Dako (Glostrup, Denmark). A mouse monoclonal antibody against P75 was purchased from Diagnostic BioSystems (Pleasanton, CA, USA).

\section{Enzyme immunohistochemistry}

Immunohistochemical experiments were performed using the EnVision/HRP system (Dako) as detailed previously (Al-Eryani, 2013). After deparaffinization, sections were autoclaved in citrate buffer $(\mathrm{pH} \mathrm{6.0)}$ at $121^{\circ} \mathrm{C}$ for $10 \mathrm{~min}$, to restore antigenicities of $\mathrm{CK} 13$, CK17 and P75. For control studies on the antibodies, the primary antibodies were replaced with preimmune rabbit or mouse IgGs.

\section{Results}

Tissue morphologicalfeatures of GCT-PCH as compared to SCC

In granular cell tumor (Figure 1a) the pseudocarcinomatous hyperplastic epithelium covering the granular cell tumor may easily be mistaken with
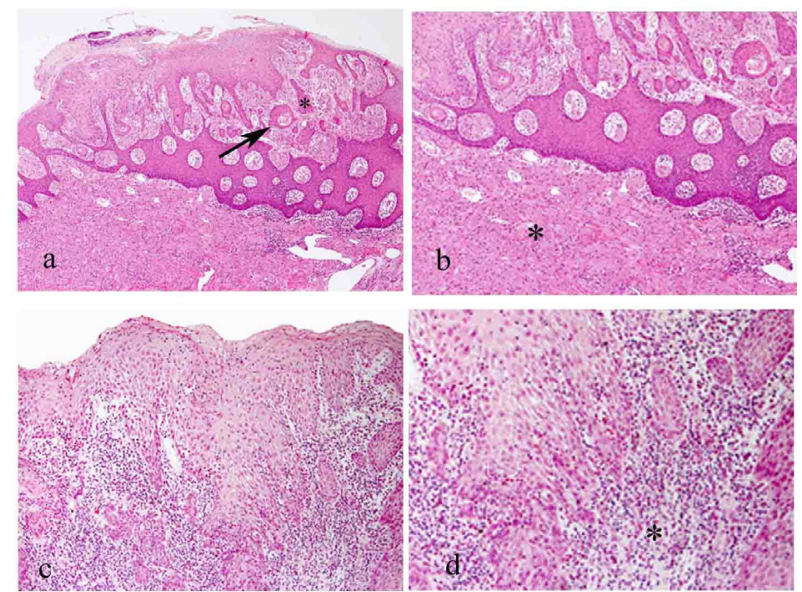

Figure 1. Morphological Features of the Granular cell Carcinoma (GCT) (a, b); In Comparison to Squamous Cell Carcinoma (SCC) (c, d). HE stain (a-d); (a, c) $\times 40$, $(\mathrm{b}, \mathrm{d}) \times 80$. In GCT (a) the pseudocarcinomatous hyperplastic epithelium covering the GCT may easily be mistaken with SCC. Both pseudoepitheliomatous hyperplastic epithelial island (asterisk) and keratin pearls (arrow) are mimicking the invasive SCC (c). (b) An area from (a) at a higher magnification that highlights the connective tissue difference between GCT and SCC (d). Note the connective tissue in (b) is composed mainly from polygonal cells with abundant granular cytoplasm (asterisk) (should be replaced with higher magnification), while in the SCC counterpart (d) the connective tissue is composed mainly from basophilic inflammatory cells (asterisk) squamous cell carcinoma. Tissue architectural features such as cells nests (asterisk) and keratin pearls (arrow) mimic invasive SCC (Figure 1c). However, connective tissue differences between GCT and SCC are evident. The connective tissue in GCT (Figure 1b) is composed mainly from polygonal cells with abundant granular cytoplasm (asterisk) gives the connective tissue an eosinophilic appearance, while in SCC (Figure 1d) the connective tissue may have an abundant number of inflammatory cells (asterisk) and thus a basophilic background, and an absence of polygonal cells with granular cytoplasm. These

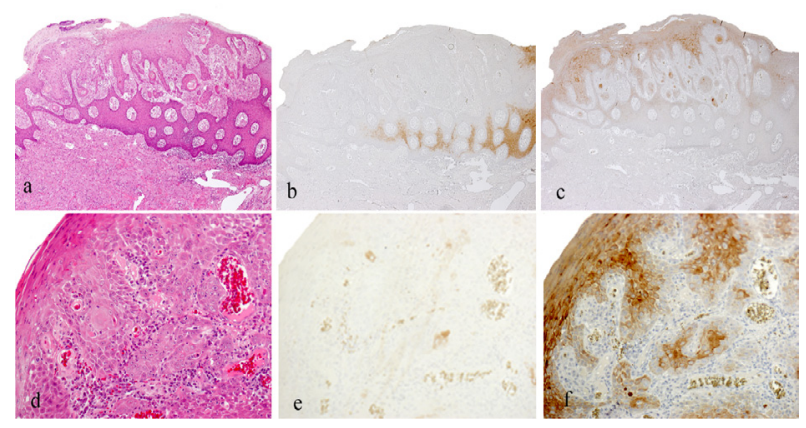

Figure 2. Immunohistochemical Stains Demonstrate Cytokeratin Profile of the Pseudoepitheliomatous Hyperplasia Associated with the GCT. HE stains (a, d); and immunoperoxidase stains for CK13 (b, e), CK17 (c, f), hematoxylin counterstain; $\times 40$. HE stains in (a) show two histologically distinguishable epithelia overlying the GCT. The upper (asterisk) epithelium is pseudocarcinomatous hyperplastic epithelium with evident irregular rete ridges, keratin pearls and invasive-like epithelium nests. In the same specimen, normal epithelial tissue is noted (arrow head). In (b) CK13+ cells were only found in the normal epithelia area while CK17 immunopositivities were restricted to SCC-like part which contained the irregular rete ridges, invasion-like nests and keratin pearls (c). In (d) HE stain shows oral SCC, (e) K13 is not expressed in the scc cancer cells; however, in (f) cancer cells are immunopositive for $\mathrm{K} 17$

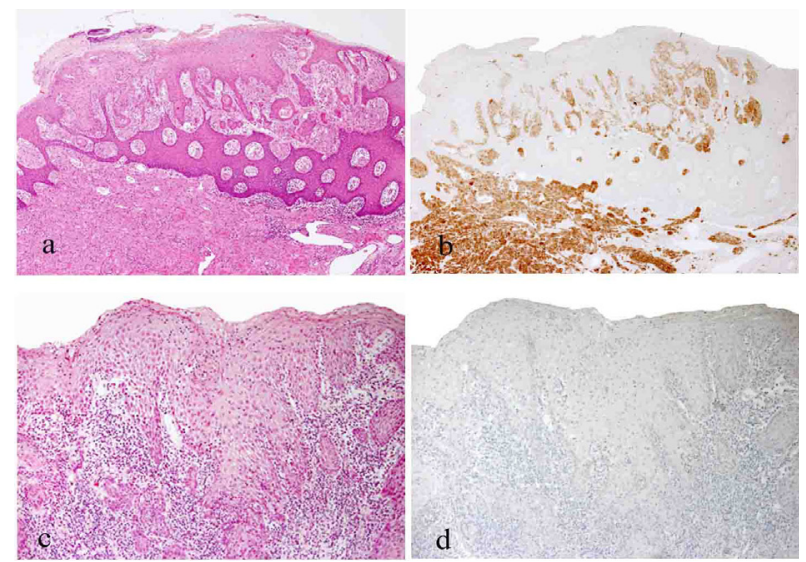

Figure 3. P75 Immunohistochemical Stains for Both Granular Cell Tumor (a, b) and Squamous Cell Carcinoma (c, d). HE stains (a, c), and immunoperoxidase stains for P75 (b, d), hematoxylin counterstain, $\times 40$. HE stains show granular cell tumor in (a) and squamous cell carcinoma in (c). Lesional cells in granular cell tumors demonstrate positive immunoreaction for p75 (b), while the cells were not positive in squamous cell carcinoma tissue (d) 
results show that while the $\mathrm{PCH}$ may be diagnostically challenging, the connective tissue findings are the most useful and simple way to distinguish between these two entities.

\section{IHC stains demonstrate cytokeratin profile of $\mathrm{PCH}$ associated with GCT}

To understand the cytokeratin pattern of the GCT associated $\mathrm{PCH}$, we compared the keratin staining pattern of the pseudocarcinomatous hyperplastic epithelium which has irregular rete ridges, keratin pearls and invasive-like cell nests (Figure 2a; asterisk) to the normal epithelium overlying GCT (Figure 2a; arrow head) and oral SCC (Figure 2d). CK13+ cells were only found in the normal epithelium area (Figure 2b). While in (Figure 2c), CK17 immunopositivity was restricted to the SCClike part. This result shows reciprocal expressions of CK17 and CK13 between the pseudocarcinomatous and normal epithelia. This reciprocal pattern is typically the cytokeratin pattern for oral SCC (Figure 2e, f). These results show the similarity in IHC profile between $\mathrm{PCH}$ and SCC.All the cases represent the same staining pattern.

\section{P75 IHC stains for both GCT and SCC}

Lesional cells in granular cell tumors demonstrate positive immunoreaction for p75 (Figure 3b), however, in SCC, neither cancer cells nor the connective tissue is positive for p75 (Figure 3d). These results show that P75 stain is a very useful method to distinguish between GCT and SCC. All the cases represent the same staining pattern.

\section{Discussion}

The aim of this work was to simplify discrimination between GCT-PCH and oral SCC. That was achieved by emphasizing several findings: 1 ) It is very difficult to distinguish morphologically between oral SCC and GCT-PCH without consideration of the under lying connective tissue, 2) IHC stains for $\mathrm{PCH}$ to differentiate it from oral SCC might be misleading, and 3) IHC for GCT (the connective tissue component) is a better guide for accurate diagnosis.

We showed that SCC and $\mathrm{PCH}$ have common morphological features such as keratin pearls and carcinoma-like invasive islands. Moreover, other studies have showed that $\mathrm{PCH}$ contains numerous mitotic figures and mildly atypical nuclei (Wolber, et al. 1991; Abu-Eid and Landini 2006). Therefore, $\mathrm{PCH}$ is nearly indistinguishable from infiltrative SCC; however, the underlying connective tissue is the essential part for distinguishing between the two entities.

Although many studies have claimed that CK13 $(-) / C K 17(+)$ is the unique profile pattern of oral SCC (Mikami, Cheng et al. 2011 ; Kitamura, Toyoshima et al. 2012 ), we showed that CKs pattern for GCT-PCH is CK13 (-) /CK17 (+). CK is just a one example of the possible IHC similarity between PCH and oral SCC. Therefore, we highly recommend reassessment of the many published case reports that claim the malignancy of GCT-PCH or the association between GCT and oral SCC based on IHC alone.
Because it is believed that Granular cell tumor histogenesis is derived from neural origin (Miettinen, et al. 1984), we suggest P75 as an excellent candidate to stain GCT. Many studies have showed that $98 \%$ of GCT is immunopositive for P75 which makes this stain useful for identifying GCT (Vered et al., 2009).

Finally, we suggest that making a correct diagnosis of GCT should be predicated on knowledge of GCT features, its associated PCH morphology and also IHC when needed. In addition, more studies on the molecular biology of $\mathrm{PCH}$ to answer the question of how $\mathrm{PCH}$ is able to mimic oral SCC without carrying its malignant phenotype are needed.

\section{References}

Abu-Eid R, Landini G (2006). Morphometrical differences between pseudo-epitheliomatous hyperplasia in granular cell tumours and squamous cell carcinomas. Histopathol, 48, 407-16.

Al-Eryani K, Cheng J, Abé T, et al (2013). Hemophagocytosismediated keratinization in oral carcinoma in-situ and squamous cell carcinoma: a possible histopathogenesis of keratin pearls. J Cell Physiol, 228, 1977-88.

Freitas VS, dos Santos JN, Oliveira MC, et al (2012). Intraoral granular cell tumors: clinicopathologic and immunohistochemical study. Quintessence Int, 43, 135-42.

Huyskens J, Geniets C (2014). Granular cell tumor in the breast mimicking breast carcinoma. JBR-BTR, 97, 289-90.

Kitamura R, Toyoshima R, Tanaka H, et al (2012). Association of cytokeratin 17 expression with differentiation in oral squamous cell carcinoma. J Cancer Res Clin Oncol, 138, 1299-310.

Lack EE, Worsham GF, Callihan MD, et al (1980). Granular cell tumor: a clinicopathologic study of 110 patients. J Surg Oncol, 13, 301-16.

Miettinen M, Lehtonen E, Lehtola H, et al (1984). Histogenesis of granular cell tumour--an immunohistochemical and ultrastructural study. J Pathol, 142, 221-9.

Mikami T, Cheng J, Maruyama S, et al (2011). Emergence of keratin 17 vs. loss of keratin 13: their reciprocal immunohistochemical profiles in oral carcinoma in situ. Oral Oncol, 47, 497-503.

Neville BW, Damm DD, Allen CM, et al (2009). Granular cell tumor. in "oral \& maxillofacial pathology". $3^{\text {rd }}$ ed. Philadelphia, Pa, WB Saunders Co: 536-7.

Patti R, Almasio PL, Di Vita G (2006). Granular cell tumor of stomach: a case report and review of literature. World $J$ Gastroenterol, 12, 3442-5.

Tan KB, Tan SH, Aw DC, et al. (2013). Simulators of squamous cell carcinoma of the skin: diagnostic challenges on small biopsies and clinicopathological correlation. J Skin Cancer, 2013, 752864.

Vered M, Carpenter WM, Buchner A (2009). Granular cell tumor of the oral cavity: updated immunohistochemical profile. $J$ Oral Pathol Med, 38, 150-9.

Wolber RA, Talerman A, Wilkinson EJ, et al (1991). Vulvar granular cell tumors with pseudocarcinomatous hyperplasia: a comparative analysis with well-differentiated squamous carcinoma. Int J Gynecol Pathol, 10, 59-66.

Zhang M, Sun ZQ, Zou XP (2014 ). Esophageal granular cell tumor: Clinical, endoscopic and histological features of 19 cases. Oncol Lett, 8, 551-5. 